\title{
Registration Center Service
}

National Cancer Institute

\section{Source}

National Cancer Institute. Registration Center Service. NCI Thesaurus. Code C93359.

The service of recording subject participation on a study. The service may include

allocation to an Arm or a portion of an Arm (when secondary allocations may occur). 